\title{
Air Entrainment and Dust Generation from a Falling Stream of Bulk Material ${ }^{\dagger}$
}

\author{
Paul Cooper and Peter C Arnold \\ Department of Mechanical Engineering \\ University of Wollongong*
}

\begin{abstract}
Bulk materials handling operations involving falling streams of the bulk material are common throughout industry. Proper design of any fugitive dust control system servicing such operations requires knowledge of the behaviour of the free-falling stream in terms of the air entrained by the falling material and the concentration of dust liberated. In this paper results of experiments on alumina powder free-falling from a hopper are presented. It was found that the material stream initially contracts in cross-section as it accelerates and a boundary layer of dusty air develops around the core stream of bulk material. Results of air entrainment per unit mass of parent material are also given. The authors describe a simple analytical model of the complex air entrainment process by treating the falling stream of material as a negatively buoyant plume of dusty air fully miscible with the ambient air. The experimental data are successfully correlated using this approach. The plume model provides a more realistic description for the situation considered here than previous theoretical treatments based on air movement induced by isolated particles independently falling through quiescent air.
\end{abstract}

\section{Introduction}
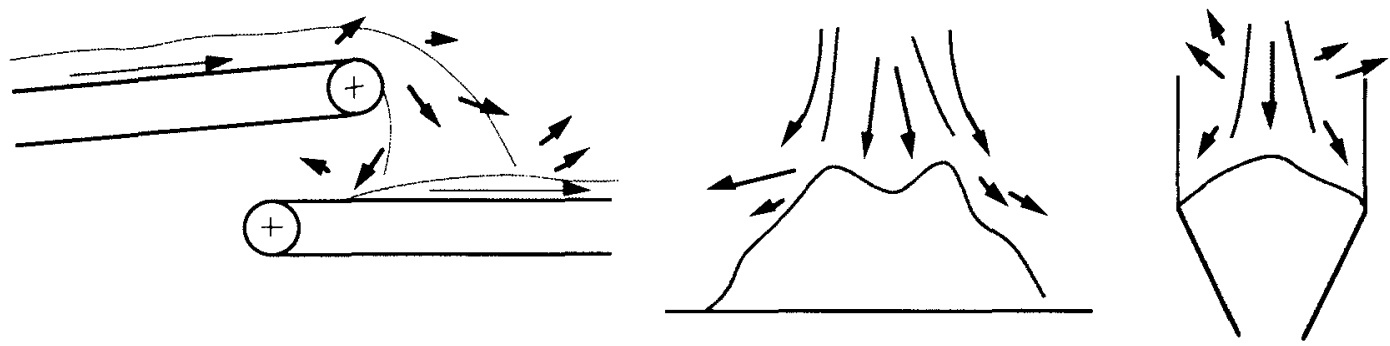

Fig. 1. Schematic of typical bulk material handling operations involving air entrainment and fugitive dust generation from falling streams of material.

Dust generation from materials handling operations has been a significant issue for many years for industrial processes as diverse as mining and food processing. However, in recent times control of dust emissions has become of increasing concern as worker exposure limits and plant emission controls have become more stringent and as recovery of valuable product previously lost in fugitive dust is seen as economically important. The cost of cleaning up plant affected by fugitive dust can be enormous. In the UK, for example, it has been estimated that annual costs

* Wollongong NSW 2522 AUSTRALIA

$\uparrow$ Received 15 June, 1995 to industry of dust, mess and spillage from materials handling operations is of the order of US\$300 million p.a. at 1987 prices (Wilkinson et al, 1989).

In a great many bulk material handling operations the material concerned undergoes a freefall and subsequent impact either on a solid surface or on the top of bulk material as in the examples of conveyor transfers and silo filling (see Figure 1). This paper deals with some of the fundamental fluid dynamic processes involved in dust generation and air movement when this type of freefall operation occurs. In all types of handling operation fugitive dust is liberated the bulk material by the process 
known as "pulvation" where the aerodynamic forces acting on individual dust particles are greater than the forces acting to maintain the cohesiveness of the bulk material. The theoretical treatment of pulvation in common freefall operations has been very limited in past literature and little quantitative data is available to designers of industrial ventilation systems on the quantity of dust liberated for a given process and bulk material or on the nature of air movement that carries away the dust.

There are several mechanisms by which dust is liberated from the parent material during operations involving freefall. In these situations all the bulk material becomes airborne and in so doing accelerates downwards and expands, entraining air and liberating fugitive dust. The flow phenomena are complex and parameters of prime importance to the design of any dust control system that is applied to control of dust include:

- air entrainment volume (and hence the required air extraction rate by an industrial ventilation system)

- dust concentration in the contaminated air

- location of the extraction point to minimise dust loading.

A schematic example of the situation of interest here is shown in Figure 2 where a falling stream of dusty material impacts on a stockpile. Fugitive dust control is effected by surrounding the process with an enclosure (this may be the silo or bin for the material concerned). To prevent dust escaping from the entry to the container, sufficient air must be extracted by

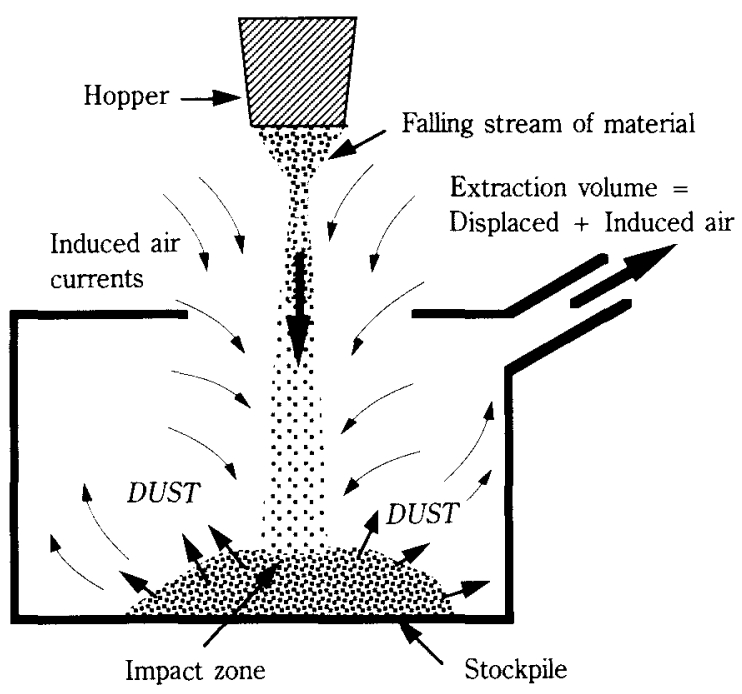

Fig. 2. Schematic of a dust control system operating on a container being filled by a falling stream of material the dust collection system to match the air influx in the stream of material (entrained air) and the air displaced by the entering solid particles (displaced air). To ensure the dust control system is designed properly, therefore, we need to know the amount of air entrained by the free-falling material. Here only the region where the material is free-falling is of concern.

When considering dust generation there are two principal physical mechanisms whereby dust leaves the bulk material and is carried away from the stockpile by air currents: i) dust is liberated during the free-fall of the parent material; ii) the falling stream impacts on the stockpile releasing the entrained air which causes pulvation of fugitive dust from the stockpile.

A great deal of research has been carried out on methods by which the "dustiness" of a given material may be determined. The general approach has been to develop a particular type of apparatus which generates dust in a repeatable manner either by dropping a known mass of the parent material in a single charge (e.g. Heitbrink et al, 1992) or by agitating the material under steady state conditions in a sealed chamber, for example. However, the "dustiness" of a material determined by these methods may not be quantitatively useful when a designer is concerned with a dust generation process that differs significantly from the dustiness test used. In the view of the present authors the most appropriate direction of research in this area is to determine dust generation from a given industrial process by considering both the characteristics of the bulk material and dynamics specific to the process concerned.

Previous fundamental research on the air entrainment and dust liberation processes from falling streams of material is limited to a very few studies. Hemeon (1962), for example, developed a means of predicting air entrainment based on the air movement induced by a single particle falling through quiescent air. As we discuss below, this approach has several limitations when used to model a falling stream of bulk material where the vast majority of particles do not behave as if they are falling through quiescent air. Very little else by way of quantitative guidance is available in the literature to the designer seeking to predict the air entrainment and dust loading on an industrial ventilation system venting an enclosure such as that shown in Figure 2. The Industrial Ventilation Manual (ACGIH, 1986), for example, gives recommended extraction rates for conveyor transfer enclosures but contains little advice as to the effect of 


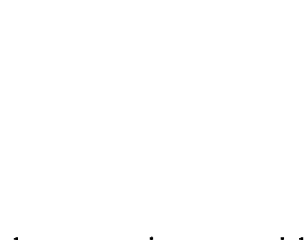

maoerialwproperties on either the required extract volumes or dust loading. To quote the Industrial Ventilation Manual on conveyor belt ventilation design (Figure VS-50-20) "Note: Dry, very dusty materials may require exhaust flow rates 1.5 to 2.0 times stated values". This clearly illustrates the qualitative nature of the published design criteria. The Industrial Ventilation Manual provides useful practical guidance on issues such as the clearance between belt loads and sealing skirting but gives no information on the likely behaviour of the bulk material and dusty air within the enclosure.

More recently Plinke et al. (1991) have performed an extensive experimental study of dust generation from falling streams of sand, cement, flour and limestone. They reported correlation of the measured dust generation as a fraction of total parent material flow rate in terms of parameters including, drop height, mass flow rate, particle size and moisture content.

The aim of our research at the University of Wollongong is to develop a theoretical model of the processes of dust generation and air entrainment to enable air entrainment and dust loading to be determined as a function of the properties of the bulk material (particle size distribution, particle density, etc) and the material handling process parameters (such as drop height and mass flow rate). The major focus of the work reported in this paper is on our progress toward quantification of the air entrainment in a falling stream of dusty bulk material.

\section{Experimental Apparatus}

A laboratory-scale experimental rig was built to carry out fundamental research on the type of situation shown in Figure 2 where a continuous stream of material enters a container through an opening in the upper surface and fugitive dust is prevented from escaping by extracting air through a second opening in the container. The experimental apparatus is shown schematically in Figures $\mathbf{3}$ and $\mathbf{4}$ and is described in detail by Smithers (1994). A double hopper arrangement was used to generate a falling stream of material with constant mass flow rate. The entire hopper system was suspended from a frame so that the mass flow rate of material could be measured directly by means of load cells attached to the suspension cables. An open-top, glass-sided enclosure, $1.0 \mathrm{~m}$ square in plan and $0.8 \mathrm{~m}$ tall, was used as the observation chamber to allow visual observation and filming of the particulate and fluid dynamic processes.

The mass flow of material was controlled by varying

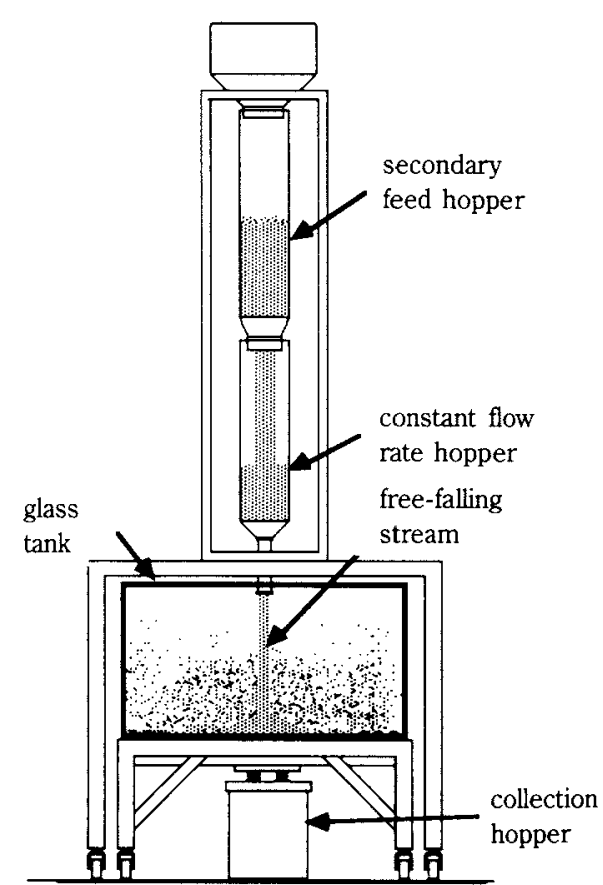

Fig. 3. Experimental apparatus for measurement of dust generation and air entrainment.

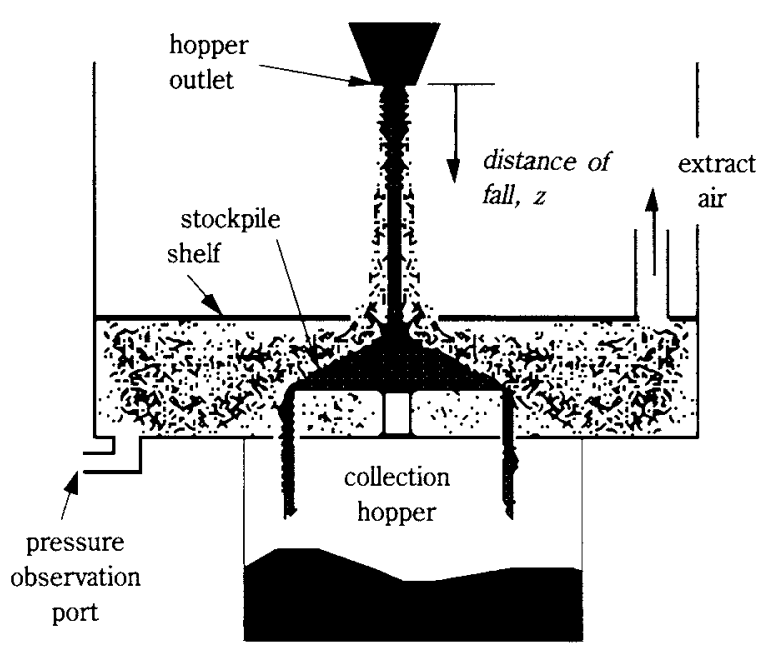

Fig. 4. Details of experimental apparatus for measurement of air entrainment.

the outlet diameter of the lower hopper. To ensure a constant drop height during the course of an experiment, stockpile height was maintained by means of a disc suspended above the chamber floor onto which 


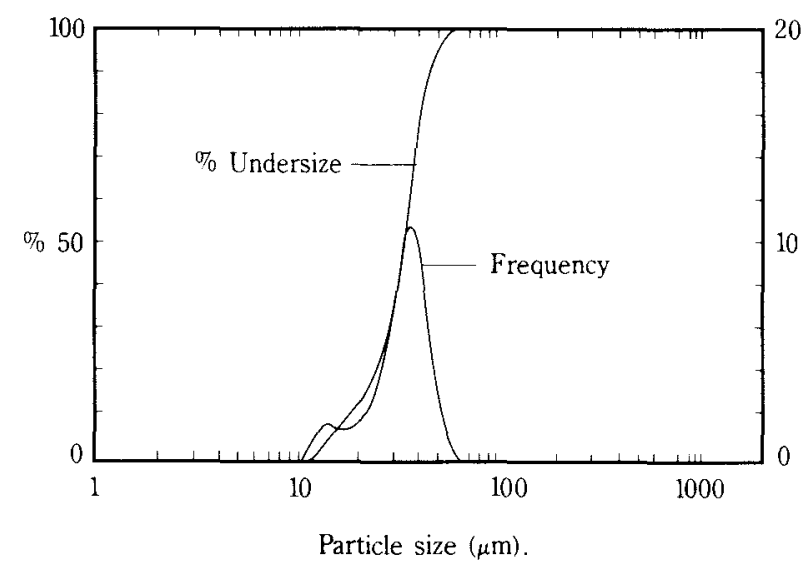

Fig. 5. Particle size distribution for alumina parent bulk material.

the material impacted. Excess material flowed off the stockpile into a collection bin below the observation chamber through an annular outlet as shown in Figure 3. The material used for the experimental work reported here was alumina. The bulk material particle size distribution was determined using a Malvern ${ }^{\mathrm{TM}}$ Series 2600 laser particle sizing system and is shown in Figure 5.

To determine the amount of air entrained in the falling material stream a shelf was placed in the observation chamber as shown in Figure 4. The falling stream of material passed through a $57 \mathrm{~mm}$ diameter hole in the enclosure shelf which was located $40 \mathrm{~mm}$ above the top of the stockpile. Air was then extracted from the region under the shelf until there was no static pressure difference between this region and outside. An extremely sensitive means of detecting when internal and external pressures were equal was provided through smoke being introduced into a small "pressure observation port" in the floor of the observation chamber. Smoke movement either in or out of the port then indicated an imbalance between internal and external pressures. The air extraction volume was measured by means of a rotameter.

\section{Experimental Results}

\subsection{Cross Sectional Area of Falling Stream of Dusty Material}

The experimental apparatus was designed for investigation of a steady state flow of material with as little disturbance to the stream on leaving the hopper as possible. The flow was generally observed to be steady with occasional disturbances lasting a fraction of a second as flow in the hopper outlet adjusted to internal inhomogeneities. Contrary to some comments in the literature, the cross-sectional area of the falling stream of material was actually found to decrease upon exiting the hopper outlet much as a viscous liquid stream diameter decreases upon release from an opening in a horizontal surface. Due to the turbulent air flow around the stream of material a "boundary layer" of dust-laden air developed around the main core region and this did increase in crosssection with increasing drop height. This "boundary layer" was the region of quiescent air affected by the downward motion of the material core stream and was limited in extent. Using smoke injected into the enclosure as a means of fluid flow visualisation,

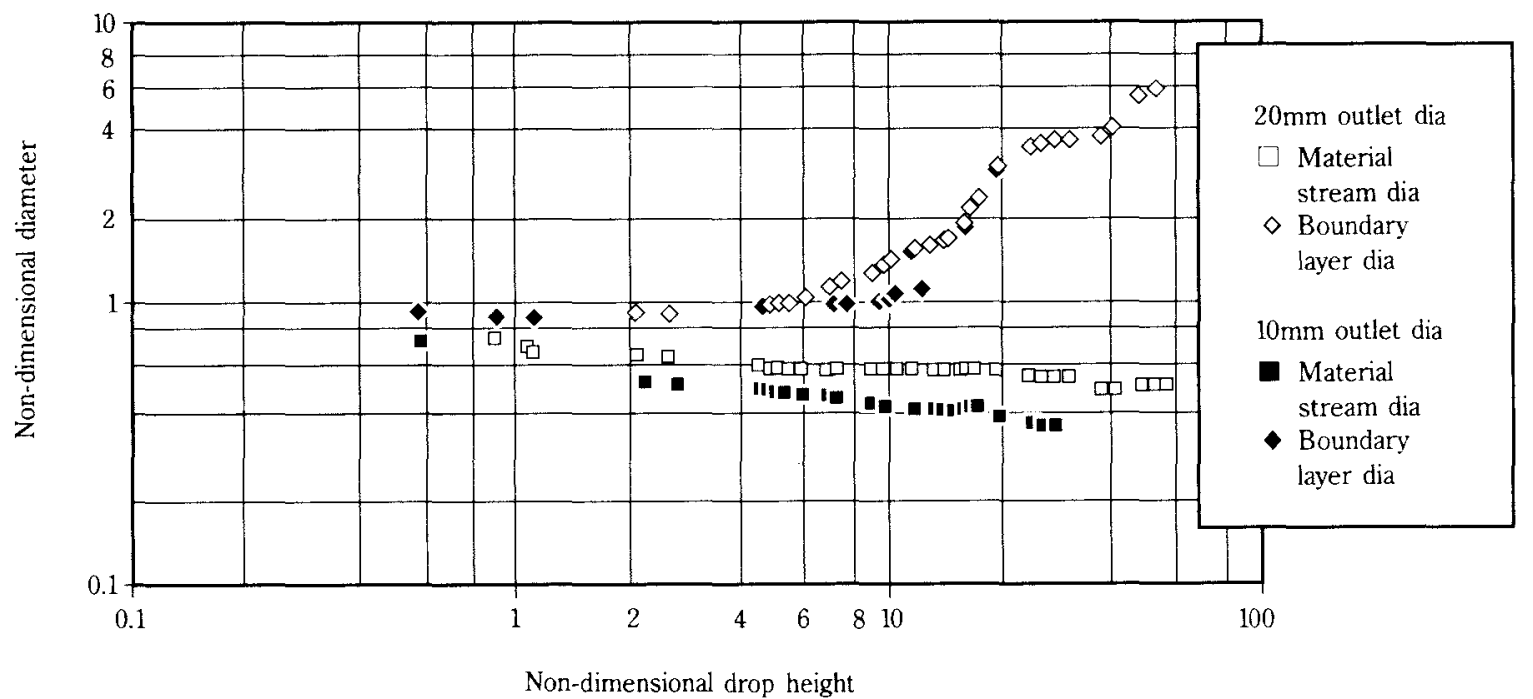

Fig. 6. Diameters of core bulk material stream and dust laden boundary layer for stream of alumina normalised against hopper outlet diameters of $10 \mathrm{~mm}$ and $20 \mathrm{~mm}$ (Woodall, 1993). 
the ofiameter of the boundary layer was estimated o(Woodall, 1993). As the distance, $z$, through which the material stream fell increased, so the turbulent motion of air and smaller particles led to a blurring of the interface between the falling stream and the "boundary layer". However, the boundary of the main core of bulk material remained distinct over the drop heights investigated to date (i.e. up to $830 \mathrm{~mm}$ ). Quantitative experimental results of the variation of material stream and boundary layer cross-section with drop height are shown in Figure 6 where drop height and the diameters of the core stream of material and of the boundary layer are non-dimensionalised with respect to the hopper outlet diameter.

\subsection{Dust Generation from the Falling Stream}

Two distinct dust generation mechanisms were observed as described above: i) dust liberation within the falling stream and ii) dust liberation as the falling stream impacted on the stockpile. To determine how dust generation in this first region is related to drop height and particle size a MetOne ${ }^{\mathrm{TM}}$ dust particle counter was used to measure the dust concentration in the experimental enclosure above the shelf. The data shown in Figure 7 are necessarily qualitative in nature since it was not possible to sample isokinetically due to difficulties in matching local air flow rates in the experimental chamber to the air sampling extraction rate. However, the data presented provide a useful insight into the way in which dust generation is affected by both drop height and dust particle size. Further research into this aspect of the problem is ongoing.

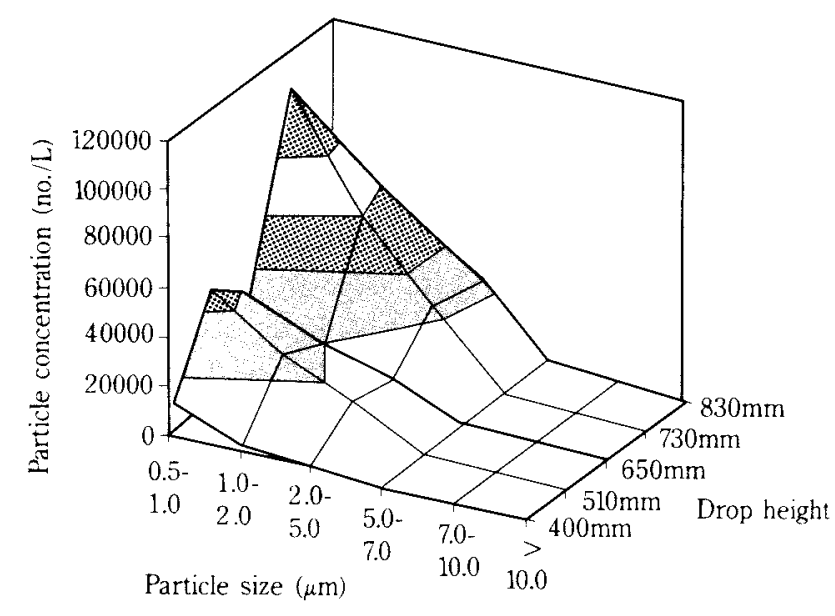

Fig. 7. Alumina dust concentration measured above shelf for $\phi 10 \mathrm{~mm}$ hopper outlet.

\subsection{Air Entrainment by the Falling Stream of Material}

A number of experiments were carried out to determine the dependence of air entrainment on drop height and mass flow rate of the alumina. The influence of drop height on air entrainment is clearly very important in design of materials handling operations such as conveyor transfers. Air entrainment flow rates, $Q_{\text {ent }}$, per unit mass flow, $\dot{m}$, of the parent bulk material are shown in Figure 8 for a constant hopper outlet diameter of $10 \mathrm{~mm}$. Here $Q_{\text {ent }}$ is the total air flow extracted from beneath the shelf of Figure 4 less the volume flow of the parent material. The results from twelve individual experiments are presented in Figure 8 and air entrainment, $Q_{e n t}$, is seen to be proportional to drop height raised to the power 1.8 .

The relationship between air entrainment per unit mass flow to actual mass flow is important in determining the validity of any theoretical model of the entrainment process. Our experiments confirmed the fact that more air is entrained as the mass flow rate of the bulk material increases. However, the amount of air entrained per unit mass of material, $Q_{\text {ent }} /(\dot{m})$, decreases with increasing mass flow rate as shown in Figure 9. This is an important finding as this indicates that as the bulk material mass flow rate increases, so the air entrainment by each individual particle decreases. This result demonstrates that air entrainment models based on the behaviour of individual particles in quiescent air cannot model the influence of bulk material mass flow rate on entrainment satisfactorily.

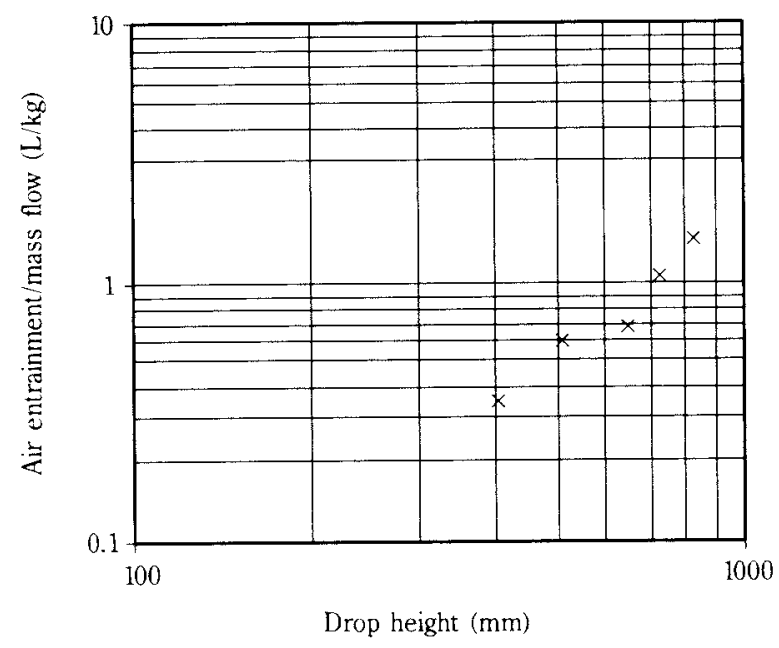

Fig. 8. Air entrainment normalised to parent bulk material (alumina) flow rate versus drop height for $\phi 10 \mathrm{~mm}$ hopper outlet. 


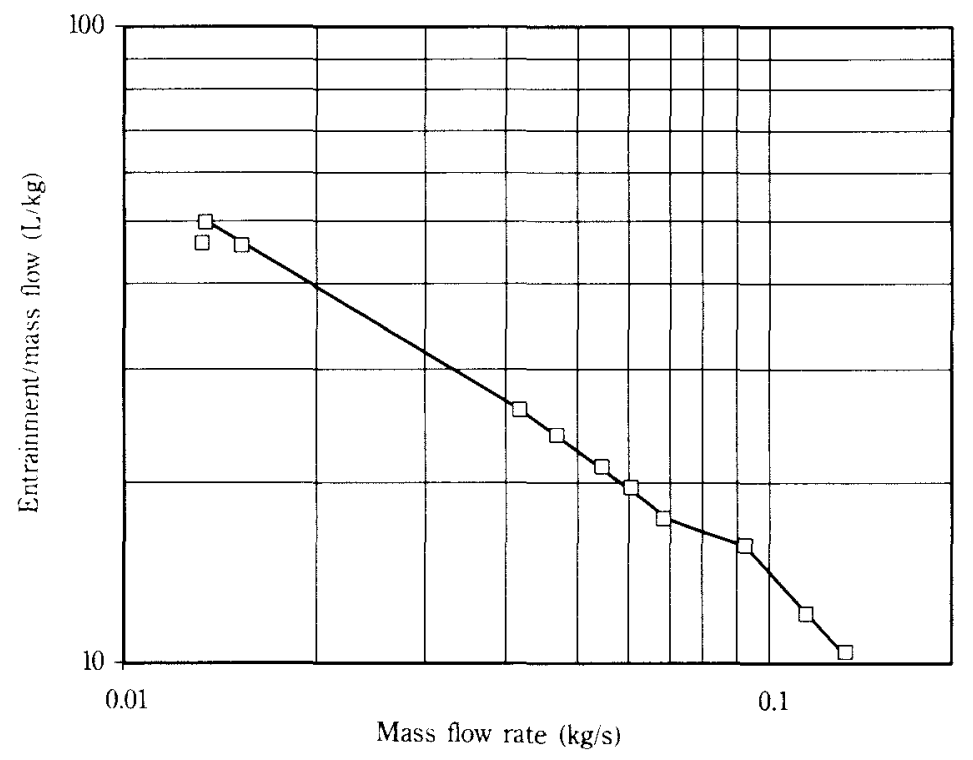

Fig. 9. Air entrainment normalised with respect to parent material flow rate for constant drop height, $z=650 \mathrm{~mm}$.

\section{Analytical Models of Air Entrainment}

The movement of individual particles within the falling stream of any bulk material will be extremely complex. The motion of an individual particle in quiescent air is in itself a complex phenomenon where drag on the particle changes as the particle accelerates and if the particle is sufficiently massive it will attain a velocity such that turbulent flow is induced in its wake. One might therefore visualise the air and particle motion in a falling stream of

work done by drag forces of a falling particle accelerated from rest to terminal velocity in quiescent air

For a continuous stream of falling material, the drag forces were assumed to be a multiple of that arising from a single particle falling through quiescent air. Hemeon's theory essentially treated the stream of bulk material as behaving in the same way as multiple single particles and has been used to predict the volumetric flowrate of air, $Q_{i n d}$, induced by a stream of material of cross-section area, A. In SI units this flow rate may be shown to be (Smithers, 1994):

$$
Q_{\text {ind }}={\frac{0.66 \mathrm{~g} \dot{m}(h A)^{2}}{d_{p} \rho_{p}}}^{1 / 3}
$$

Where $d_{p}$ and $p_{p}$ are the particle diameter in metres and density in $\mathrm{kg} / \mathrm{m}^{3}$, respectively, $g$ is the gravitational acceleration constant, $\dot{m}$ is bulk material mass many particles of different sizes to be influenced by both the macroscopic flow field induced by the material falling under the influence of gravity and by the small scale turbulent motion induced by the motion of individual particles.

The first attempt to model this complex situation with regard to prediction of air entrainment by the falling stream was reported by Hemeon (1962). Hemeon's method of predicting the air volume entrained in a falling stream of material is based on the following assumption.

work needed to accelerate the entrained air from rest to entrained velocity

flow rate in $\mathrm{kg} / \mathrm{s}$ and $\mathrm{h}$ is the drop height in metres. (Note that several major assumptions are made in the course of the derivation of equation (1) in regard to the drag on the particles in the ambient air). The value of $Q_{\text {ind }}$ obtained is then modified by an arbitrary factor to account for the fact that the material stream does not behave as individual particles falling through quiescent air. Hemeon's model has also been used by others such as Morrison (1971) and Tooker (1992) to predict the air flow through an opening induced by a falling stream of material.

While the approach adopted by Hemeon has been quoted in the literature many times there are several problems associated with this model of the air entrainment process which include: a) a continuous stream of material certainly does not behave in the 
sanpe way as individual particles falling independently through quiescent air-particles will reach much higher velocities than if acting individually in quiescent air, turbulent wakes downstream of the individual particles will increase turbulence levels and influence the motion of particles following, etc; b) most bulk materials have a wide range of particle sizes and yet a single value of $d_{p}$ must be chosen in equation (1); c) there is no way of determining the cross section of the stream, A, at a given point unless the process is already in place and observable.

The theoretical approach adopted by the present authors is to treat the falling stream as a collection of particles. The behaviour of the stream is actually dependent on many factors but as a first approximation one may take it to posses characteristics from two extremes, firstly, where the stream is made up of very massive particles that fall vertically under the influence of gravity alone as shown in Figure 10a (negligible drag forces) and, secondly, where the stream is made up of extremely fine particles with no cohesion that fall in a plume as shown in Figure $10 \mathrm{~b}$ which is analogous to the way in which a miscible plume of buoyant hot air rises from a smoke stack.

Consider first the extreme situation shown in Figure 10a where a bulk material of massive particles falls from a hopper. A steady flow might be regarded as falling with very little drag force acting on the majority of the particles in the stream and that all particles accelerate at a rate equal to the gravitational constant, $g$. If one assumes that the momentum of the entrained air is negligible compared to that of the solid particles within the stream and that the slip velocity between the air and particles is very small, then the air entrained may be calculated simply according to the principle of conservation of volume; i.e. the entrained air volume flow rate must be equal to the cross-sectional area of the stream multiplied by the difference in velocity at the end of the drop-height in question and at the start of free-fall. The entrained air volumetric flow rate, $Q_{e n t}$, is then given by:

$$
Q_{\text {ent }}=\left(\left(V_{0}^{2}+2 g h\right)^{1 / 2}-V_{0}\right) A_{0}
$$

where $V_{0}$ and $A_{0}$ are the bulk material velocity and cross-sectional area at the start of free-fall, respectively, $g$ is gravitational acceleration and $h$ is the drop height.

The second and opposite extreme is the case of a stream of very fine powder falling from the outlet of a hopper. If the particles are extremely small and not cohesive in any way, their terminal velocity will be very small compared to any induced air velocities and the resulting flow would develop as a miscible plume from a source of negative buoyancy (see Figure 10b). The volumetric flow in a plume of positively or negatively buoyant fluid miscible with ambient fluid may be determined using well known plume flow equations, such as those developed by Morton et al (1956). The plume may be modelled using the "entrainment assumption" which may be

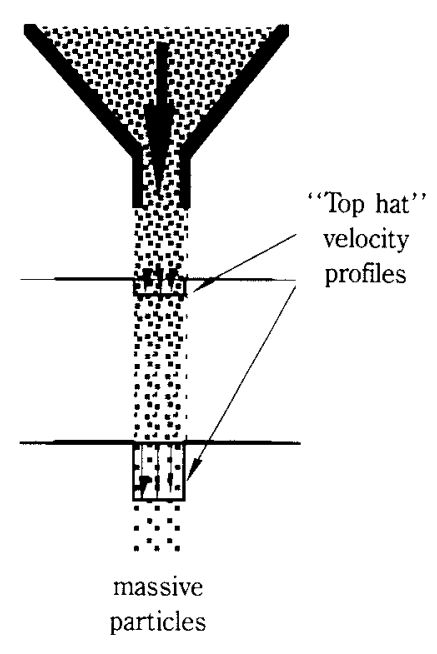

(a)

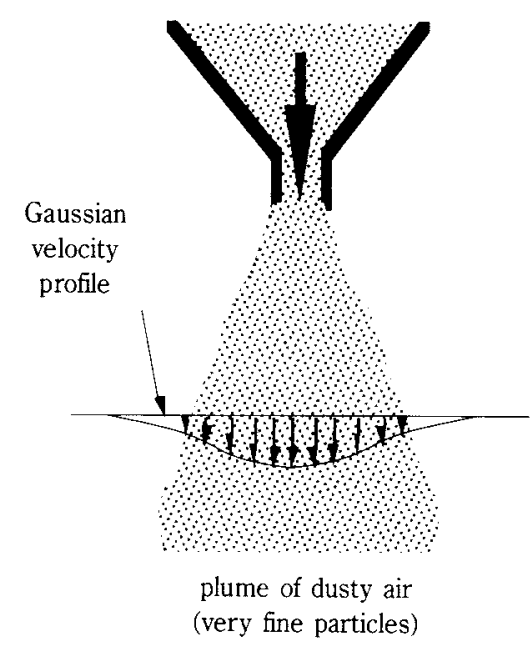

(b)

Fig. 10. Two possible extremes of free-falling particle stream behaviour: a) large particles falling vertically under gravitational forces alone b) extremely fine particles acting as a miscible plume. 
surnmarised by stating that the horizontal velocity of ambient air drawn into the plume at a given crosssection is directly proportional to the mean vertical velocity of fluid within the plume. The constant of proportionality is known as the "entrainment constant", $\alpha$. Solution of the governing equations for mass, momentum and density may be solved analytically. The volumetric flow rate, $Q_{\text {plume }}$, at a given distance $h$ from the point source (theoretically with zero mass and momentum flux) is given by the relation:

$$
Q_{\text {plume }}=C B^{1 / 3} h^{5 / 3}
$$

where $C$ is constant (usually of the order 0.1 for miscible plumes) related to the entrainment constant, $\alpha$, and $B$ is the buoyancy flux. In the situation considered here, where the fluid surrounding the plume is not significantly stratified, $B$ is constant throughout the vertical plume development and is defined as volumetric flow, $Q$, multiplied by reduced gravity $g^{\prime}=g\left(\rho-\rho_{\infty}\right) / \rho_{0}$ where $\rho$ is the mean density of the plume, $\rho_{\infty}$ is the density of the ambient fluid and $\rho_{0}$ is a reference density).

Thus, there are several ways in which the air entrainment by a stream of falling bulk material might be approximately modelled. Three such simple analytical models described above may be summarised as: the individual particle model (Hemeon); the massive particle model; the miscible plume model.

\section{Discussion}

The behaviour of the stream of bulk material leaving the hopper described in section 3.1 above indicates that the majority of the parent material falls in a stream that may in fact have a cross sectional area less than that of the hopper outlet. However, the existence of a "boundary layer" of dusty (negatively buoyant) air around this core stream indicates that air is induced to flow over a considerably wider cross section than the material stream itself. It seems likely then that from the point of view of air entrainment the stream would indeed show characteristics applicable to both the massive particle and miscible plume models.

The quantitative air entrainment results presented in Figures $\mathbf{8}$ and $\mathbf{9}$ provide a means of assessing the validity of the three models of air entrainment outlined above. As one would intuitively expect, the amount of air entrained by a falling stream of material increases with increasing drop height as indicated in Figure 8. All three models predict this in a qualitative sense. Also it is clear that as the total mass flow rate of the parent material increases so the interaction of each particle with the ambient air is lessened in the average and the air entrained per unit mass of parent material will decrease. We have also found this to be the case experimentally as shown in Figure 9 and we now demonstrate that the experimental data presented here goes someway to demonstrating that the plume analysis of air entrainment provides the best air entrainment model to date.

Assuming that air entrainment, $Q_{\text {ent }}$, per unit mass flow rate of parent material mass, $\dot{m}$, is related to drop height, h, and material mass flow rate, $\dot{m}$, by the relationship:

$$
\left(Q_{e n t} / \dot{m}\right) \approx k(\dot{m})^{m} \times h^{n}
$$

(where $m, n$ and $k$ are empirically determined constants), the three entrainment models described above in equations (1), (2) and (3) then lead to values of the exponents $m$ and $n$ as shown in Table 1. The experimental values of the exponents have been derived from our data presented in Figures $\mathbf{8}$ and 9. Clearly from this qualitative examination of the theoretical and experimental data, the plume model matches observations better than either Hemeon's approach or the simple large particle model.

The utility of the plume model is demonstrated even more clearly when it is used as a predictive tool. The air entrainment volume data for all the experiments in the present study have been correlated using equation (3) and an entrainment constant of 0.031 . Figure 11 shows a plot of air entrainment predicted using equation (3) for each set of experimental conditions, $h$ and $\dot{m}$, against the measured values of $\left(Q_{\text {ent }} / \dot{m}\right)$ in units of $\mathrm{L} / \mathrm{kg}$. The data spread is relatively small and the correlation holds over more than an order of magnitude of variation in $\left(Q_{e n t} / \dot{m}\right)$. This lends weight to our belief that application of the plume model may be extended to cover larger scale industrial situations and with appropriate calibration the model could be used as a design tool.

Table 1. Theoretical and experimental values of exponents used for determining air entrainment volume per unit mass of parent material.

\begin{tabular}{|l|c|c|}
\hline Theoretical Model & $\mathrm{2}$ Exponents \\
\hline Hemeon & -0.67 & 0.67 \\
\hline Large particle model & -1.0 & 0.5 \\
(near to source) & (far from source) \\
\hline Plume model & -0.67 & 1.67 \\
\hline Experimental results & -0.65 & 1.8 \\
\hline
\end{tabular}




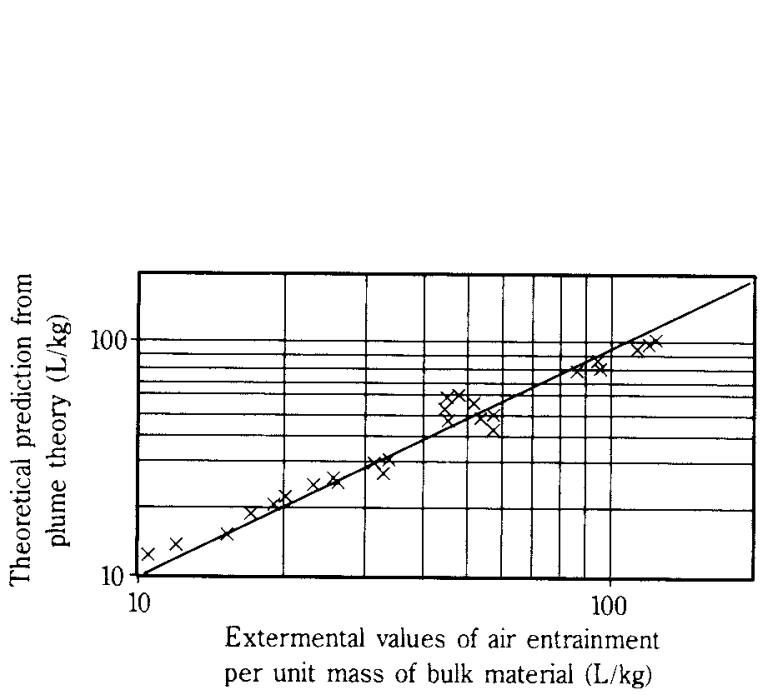

Fig. 11. Air entrainment predicted from simple plume theory against measured values (entrainment constant $=0.031$ )

The fluid dynamics analysis of conventional miscible fluid plume flow assumes either gaussian or "top-hat" velocity and density profiles across the plume at any cross-section. The velocity profile across a falling stream of bulk material is not known, however, the fact that the plume model works in the application here is not a surprise and adds to the ubiquitous success of the "entrainment assumption" in situations as diverse as volcanic eruptions, laboratory experiments and oceanographic phenomena. The entrainment constant that best correlates our data here is 0.031 . However, since this is an empirical constant further research is required to determine whether the constant is widely applicable to materials handling applications or whether it is a strong function of parameters such as particle size distribution and mass flow.

Moreover, the authors are cognisant of the fact that a large number of factors affect air entrainment which they have not dealt with here explicitly, due to lack of space. Clearly the manner in which a falling stream of material becomes airborne is important. In our research to date we have been concerned with the fundamental flow processes involved and have chosen a simple method of releasing the parent bulk material. In many practical situations the material becomes airborne with a considerable initial momentum, possibly in a horizontal direction as is the case with conveyor transfers. Similarly, the influence of nearby solid objects and boundaries may influence air flow around the falling stream and hence affect entrainment volume. These matters are currently under investigation at the University of Wollongong.

Designers of fugitive dust control enclosures involving falling streams of materials should be aware of the mechanisms by which air is entrained and dust liberated from the free-falling material and the way in which air de-entrains upon impact with the stockpile carrying significant quantities of dust with it. Know- ledge of these mechanisms allows the designer to predict more accurately the best positions for extract air removal from an enclosure to minimise the dust loading on the air cleaning system. To facilitate development of a theoretical model for accurate prediction of air entrainment and dust generation, further research is necessary to model the various two-phase flow phenomena involved, to model the influence of bulk material properties on dust generation and air entrainment and to test the theoretical models using full-scale plant.

\section{Conclusions}

Air entrainment and dust generation by falling streams of bulk materials is important in the design of many fugitive dust control systems. Currently there is little quantitative data available in the literature to guide the designer of such systems. In this paper experimental results have been presented for air entrainment and dust generation by a stream of alumina falling under steady state conditions from a small hopper.

The experimental results have demonstrated the following: the cross sectional area of the stream of bulk material decreases with increasing drop over the heights investigated in the present study-a boundary layer of dusty air of increasing cross section develops with increasing drop height; air entrainment per unit mass flow of parent material is proportional to $Z^{1.8}$, where $Z$ is distance from the hopper outlet; the air entrainment per unit mass flow of parent material decreases with increasing mass flow.

Three simple analytical models of the air entrainment process have been discussed: the single particle model of Hemeon; the massive particle model; and the miscible plume model. The miscible plume model is based on the plume equations commonly used in environmental fluid dynamics and has been shown here to provide the best correlation of the experimental air entrainment data.

\section{Nomenclature}

$A=$ cross sectional area of falling stream $[\mathrm{A}]$

$B=$ buoyancy flux $\left[\mathrm{m}^{4} / \mathrm{s}^{3}\right]$

$d_{p}=$ particle diameter $[\mathrm{m}]$

$C=$ constant in equation (3)

$g=$ gravitational acceleration constant $\left[\mathrm{m} / \mathrm{s}^{2}\right]$

$g^{\prime}=$ reduced gravity $g\left(\rho-\rho_{\infty}\right) / \rho_{0}\left[\mathrm{~m} / \mathrm{s}^{2}\right]$

$h=$ drop height $[\mathrm{m}]$

$Q=$ volume flow rate $\left[\mathrm{m}^{3} / \mathrm{s}\right]$ 
$\mathrm{Qent}_{\mathrm{ent}}=$ volume flow of entrained air $\left[\mathrm{m}^{3} / \mathrm{s}\right]$

$V_{0}=$ Velocity of bulk material leaving hopper $[\mathrm{m} / \mathrm{s}]$

$\alpha=$ entrainment constant

$\rho \quad=$ mean density of plume $\left[\mathrm{kg} / \mathrm{m}^{3}\right]$

$\rho_{p}=$ particle density $\left[\mathrm{kg} / \mathrm{m}^{3}\right]$

$\rho_{\infty}=$ density of ambient fluid $\left[\mathrm{kg} / \mathrm{m}^{3}\right]$

$\rho_{0}=$ reference density $\left[\mathrm{kg} / \mathrm{m}^{3}\right]$

\section{References}

1) ACGIH, American Conference of Governmental Industrial Hygienists, Industrial Ventilation-A Manual of Recommended Practice, 19th Ed (1986).

2) Heitbrink, W.A., P.A. Baron and K. Willeke: Am. Ind. Hyg. Assoc. J., 53(10), 617-624 (1992).

3) Hemeon, W.D.L., Plant and process ventilation, Industrial Press, N.Y (1962).

4) Morrison, J. N.: Trans. Soc. Mining Engineers, AIME, March 1971, 47-53 (1971).
5) Morton, B., G.I. Taylor and J.S. Turner: Proc. Royal Soc. London, Series A, 234A, 1-22 (1956).

6) Plinke, M.A.E., D. Leith, D.B. Holstein, and M.G. Boundy: Am. Ind. Hyg. Assoc. J., 52(12), 521-528 (1991).

7) Smithers, T, "Generation of dust from a falling stream of material", BE honours thesis, Dept. Mechanical Engineering, University of Wollongong (1994).

8) Tooker, G.E.: Bulk Solids Handling, vol. 12, no. 2, 227-232 (1992).

9) Wilkinson, H.N., A.R. Reed, and H. Wright: Bulk Solids Handling, 9, 93-97 (1989).

10) Woodall, I. "Investigation of various dust generation Mechanisms as a means of controlling industrial pollution", BE honours thesis, Dept. Mechanical Engineering, University of Wollongong (1993).

\section{Author's short biography}

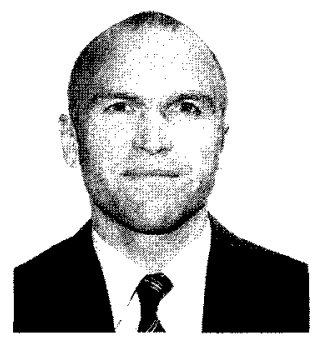

\section{Dr. Paul Cooper}

Dr. Cooper is a Senior Lecturer with the Deparetment of Mechanical Engineering at the University of Wollongong. He received his $\mathrm{BSc}, \mathrm{MSc}$ and $\mathrm{PhD}$ degrees from Imperial College (UK) and has been working in the areas of industrial ventilation and air conditioning for the past five years. His main research interests are in the fields of dust and fume control, duct network design, hot process fume control, scale modelling of fluid flows and natural convection processes.

\section{Prof. Peter Arnold}

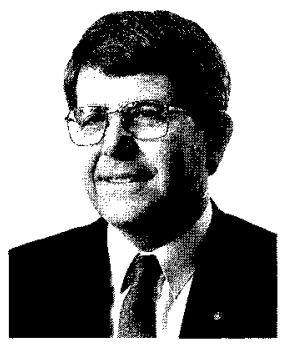

Professor Peter Arnold is Professor of Materials Handling and Processing, Department of Mechanical Engineering at the University of Wollongong and is Director of the Bulk Materials Handling Centre. In 1991 he was awarded the degree of Doctor of Science by the University of Wollongong and in 1993 was elected a Fellow of the Australian Academy of Technological Sciences and Engineering for his significant contributions to the science and technology of bulk solids handling. Professor Arnold is on the Editorial Boards for the journals Bulk Solids Handling, Powder Handling and Processing, Powder Technology as well as KONA. He is a foundation member, and currently Deputy Chairman of the Institution of Engineers, Australia, National Committee on Bulk Materials Handling. 